\title{
Evaluación del lenguaje en preescolares del norte de la ciudad de Durango, Durango, México
}

Language evaluation in preschoolers in the north of the city of Durango, Durango., Mexico

Avaliação da linguagem em pré-escolares no norte da cidade de Durango, Durango, México

Reyes-Verdín, Flor Dayana Universidad Juárez del Estado de Durango, México moon_ada@hotmail.com https://orcid.org/0000-0002-0982-0326

Ríos-Valles, José Alejandro Universidad Juárez del Estado de Durango, México alexriva@hotmail.com https://orcid.org/0000-0002-8407-3017

Salas-Name, Sagrario Lizeth Universidad Juárez del Estado de Durango, México lizeth_name@hotmail.com https://orcid.org/0000-0002-1282-626X

Soto-Rivera, Jesús Abraham Universidad Juárez del Estado de Durango, México abrahamjsr@hotmail.com https://orcid.org/0000-0001-6688-2032

Herrera-Vargas, Isela Vanessa Universidad Juárez del Estado de Durango, México Vanetch04@hotmail.com https://orcid.org/0000-0002-9154-6978 


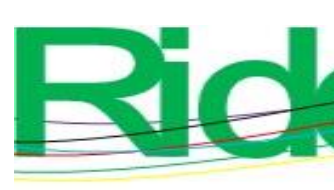

Revista Iberoamericana para la Investigación y el Desarrollo Educativo ISSN $2007-7467$

\section{Resumen}

El porcentaje de niños latinoamericanos en edad preescolar con deficiencia en el desarrollo del lenguaje oscila entre $10 \%$ y $80 \%$. Objetivo: Conocer el porcentaje de deficiencia en el lenguaje de niños preescolares del norte de la ciudad de Durango, Durango (México), para lo cual se empleó el cuestionario de madurez neuropsicológica infantil. Metodología: El estudio fue no experimental, observacional, transeccional y descriptivo, en un universo de trabajo de 833 niños, para lo que se consideró $95 \%$ de confiabilidad en una muestra estadísticamente significativa de 214 participantes; sin embargo, debido a la culminación del ciclo escolar, solo fue posible evaluar a 148 participantes en el periodo de noviembre de 2017 a junio de 2018. El grupo estudiado fue dividido en 4 subgrupos: 55-60 meses de edad, 6166 meses de edad, 67-72 meses de edad y 73-78 meses de edad.

Resultados: El alfa de Cronbach de las variables estudiadas fue 0.618. Se observaron deficiencias en las dos áreas del lenguaje evaluadas: lenguaje verbal y lenguaje no verbal. En los cuatro grupos estudiados, la mayor deficiencia se observó en el área del lenguaje verbal con porcentajes que oscilaron entre $-80.5 \%$ y $-95.9 \%$ en fluidez verbal; lenguaje expresivo con $-27.5 \%$ al $-55.2 \%$; lenguaje comprensivo con $-15.7 \%$ al $-31.1 \%$ y lenguaje articulatorio con-14.1\% al -19.3\%. Las demás variables del grupo de lenguaje no verbal —estructuración espacial, psicomotricidad, visopercepción y memoria icónica - presentaron porcentajes de deficiencia menores a los observados en las variables de lenguaje verbal, aunque se destacan atención con $-39.5 \%$ al $-43.2 \%$, ritmo con $-2.9 \%$ al $-34.5 \%$, visopercepción con $-10.6 \%$ al $-25.7 \%$, estructuración espacial con $-3.5 \%$ al $-11.1 \%$, memoria icónica con $-6.8 \%$, psicomotricidad con $-3.6 \%$. Todos los grupos de edad estudiados presentaron deficiencia en seis o más variables del instrumento de evaluación empleado. Las variables del lenguaje no verbal que presentaron resultados satisfactorios por grupo de meses de edad fueron las siguientes: estructuración espacial en el grupo de 55-60 y en el de 67-72; psicomotricidad en el grupo de 55-60, 61-66 y 67-72; visopercepción en el grupo de 55-60; memoria icónica en los grupos de 55-60 y 67-72. Conclusión: De las áreas de lenguaje evaluadas, la de mayor afectación fue lenguaje verbal, lo cual permite considerar la necesidad de implementar la evaluación del desarrollo del lenguaje en la población infantil preescolar para considerar la oportunidad de optimizarlo.

Palabras claves: lenguaje no verbal, lenguaje verbal, retraso del lenguaje. 


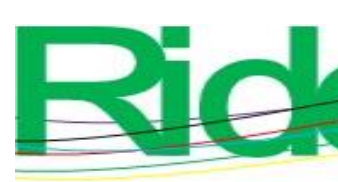

Revista Iberoamericana para la
Investigación y el Desarrollo Educativo ISSN 2007-7467

\section{Abstract}

The percentage of Latin American children of preschool age, with deficiencies in language development, ranges from $10 \%$ to $80 \%$. Objective: To know the percentage of deficiency in the language of preschool children in the north of the city of Durango, Dgo., Mexico, using the child neuropsychological maturity questionnaire. Methodology: The study was nonexperimental, observational, transectional and descriptive in a work universe of 833 children, considering a statistically significant sample of 214 participants with $95 \%$ reliability, but due to the completion of the school year it was only possible to evaluate 148 participants in a period from November 2017 to June 2018. The studied group was divided into four subgroups: a group of 55-60 months of age, second group of 61-66 months of age, third group of 67-72 months of age and the fourth group 73-78 months of age.

Results: Cronbach's Alpha of the studied variables was 0.618, observing deficiencies in the two evaluated language areas, verbal language and non-verbal language. In the four groups studied, the greatest deficiency was observed in the area of verbal language, with percentages that ranged from $-80.5 \%$ to $-95.9 \%$ in verbal fluency; expressive language with $-27.5 \%$ to $55.2 \%$; comprehensive language with $-15.7 \%$ to $-31.1 \%$ and articulatory language with $14.1 \%$ to $-19.3 \%$. The other variables of the non-verbal language group, which are spatial structuring, psychomotor skills, visual perception and iconic memory, presented lower percentages of deficiency than those observed in the verbal language variables, highlighting attention with $-39.5 \%$ to $-43.2 \%$, rhythm with $-2.9 \%$ to $-34.5 \%$, visoperception with $-10.6 \%$ to $-25.7 \%$, spatial structuring with $-3.5 \%$ to $-11.1 \%$, iconic memory with $-6.8 \%$, psychomotor skills with $-3.6 \%$. All the age groups studied showed deficiency in six or more variables of the evaluation instrument used. The non-verbal language variables that presented satisfactory results, by age group were: spatial structuring in the 55-60 group and in the 67-72 group; psychomotor skills in the 55-60, 61-66 and 67-72 group; visoperception in the 55-60 group; iconic memory in groups 55-60 and 67-72. Conclusion: The language area most affected was verbal language, which allows considering the need to implement the evaluation of language development in the preschool child population to consider the opportunity to improve the development of children's language.

Keywords: non-verbal language, verbal language, language delay. 


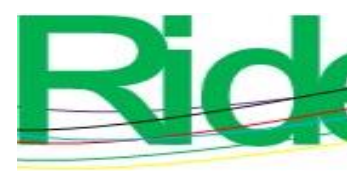

Revista Iberoamericana para la Investigación y el Desarrollo Educativo ISSN $2007-7467$

\section{Resumo}

A porcentagem de crianças pré-escolares latino-americanas com deficiências no desenvolvimento da linguagem varia de $10 \%$ a $80 \%$. Objetivo: Conhecer a porcentagem de deficiência de linguagem em crianças pré-escolares do norte da cidade de Durango, Durango (México), para as quais foi utilizado o questionário de maturidade neuropsicológica infantil. Metodologia: O estudo foi não experimental, observacional, transversal e descritivo, em um universo de trabalho de 833 crianças, para o qual foi considerada confiabilidade de $95 \%$ em uma amostra estatisticamente significativa de 214 participantes; Porém, devido ao término do ano letivo, só foi possível avaliar 148 participantes no período de novembro de 2017 a junho de 2018. O grupo estudado foi dividido em 4 subgrupos: 55-60 meses de idade, 61-66 meses , 67-72 meses de idade e 73-78 meses de idade.

Resultados: o alfa de Cronbach das variáveis estudadas foi de 0,618. Foram observadas deficiências nas duas áreas de linguagem avaliadas: linguagem verbal e linguagem não verbal. Nos quatro grupos estudados, a maior deficiência foi observada na área de linguagem verbal com percentuais que variaram entre $-80,5 \%$ e $-95,9 \%$ na fluência verbal; linguagem expressiva com $-27,5 \%$ a $-55,2 \%$; linguagem abrangente com $-15,7 \%$ a $-31,1 \%$ e linguagem articulatória com $-14,1 \%$ a $-19,3 \%$. As demais variáveis do grupo linguagem não verbal estruturação espacial, habilidades psicomotoras, percepção visual e memória icônica apresentaram percentuais de deficiência inferiores aos observados nas variáveis de linguagem verbal, embora atenção se destaque com $-39,5 \%$ a $-43,2 \%$, ritmo com $-2,9 \%$ a $34,5 \%$, percepção visual com $-10,6 \%$ a $-25,7 \%$, estruturação espacial com $-3,5 \%$ a $-11,1 \%$, memória icônica com $-6,8 \%$, habilidades psicomotoras com -3,6\%. Todas as faixas etárias estudadas apresentaram deficiência em seis ou mais variáveis do instrumento de avaliação utilizado. As variáveis de linguagem não verbal que apresentaram resultados satisfatórios por faixa etária foram: estruturação espacial no grupo 55-60 e no grupo 67-72; habilidades psicomotoras no grupo 55-60, 61-66 e 67-72; visopercepção no grupo 55-60; memória icônica nos grupos 55-60 e 67-72. Conclusão: Das áreas de linguagem avaliadas, a mais afetada foi a linguagem verbal, o que permite considerar a necessidade de implementar a avaliação do desenvolvimento da linguagem na população pré-escolar para considerar a oportunidade de otimizá-la.

Palavras-chave: linguagem não verbal, linguagem verbal, atraso de linguagem.

Fecha Recepción: Septiembre 2020

Fecha Aceptación: Febrero 2021 


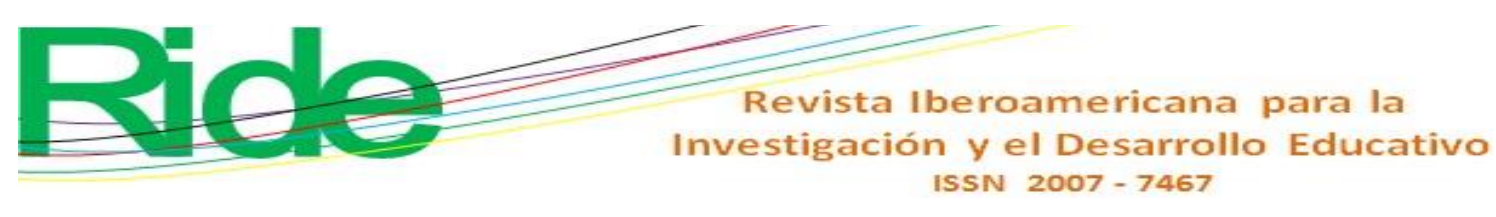

\section{Introducción}

Derivado de lo observado en diversas investigaciones, se puede señalar que entre 10 $\%$ y $80 \%$ de la población preescolar latinoamericana presenta déficit en el desarrollo del leguaje, por lo que surge la inquietud de conocer el estado de dicho problema en niños del norte de la ciudad de Durango, Durango (México).

El desarrollo del lenguaje es un proceso importante para la satisfactoria comunicación y entendimiento entre los integrantes de la sociedad, de ahí que resulte relevante evaluarlo desde las primeras etapas de la vida para propiciar las habilidades comunicativas que a su vez favorecen el desarrollo cognitivo. Según Corral (18 de mayo de 2018), "el lenguaje es la base de la comunicación del ser humano que permite expresarnos y comprender a los demás, y dependiendo de cómo lo utilicemos, vamos a construir e interpretar el mundo de manera diferente" (parr. 1).

En el niño de preescolar resulta esencial conocer el nivel del desarrollo del lenguaje, sobre todo en quienes están cercanos al inicio formal de su educación escolar, ya que se tiene conocido que la exposición y desarrollo temprano del lenguaje en estos infantes permite predecir las habilidades lingüísticas y cognitivas, así como sus consecuentes logros académicos (Romeo et al., 2018). Por eso, la identificación oportuna del nivel de desarrollo del lenguaje y comunicación oral en los niños constituye una preocupación para padres, maestros y educadores, pues de ese factor depende la evolución en la comunicación, el aprendizaje escolar y la personalidad en general (Torres, 2018).

La edad preescolar es un periodo excepcional en donde se puede aprovechar al máximo las potencialidades del niño, ya que biológicamente es una etapa en la que se establecen conexiones neuronales que constituirán la base de los procesos neurocognitivos para el aprendizaje preescolar (Escobar, 2006; Portellano, Matreos y Martínez, 2006; Romeo et al., 2018).

Son múltiples y variados los esfuerzos que en diversas partes del mundo se han realizado para identificar el nivel de desarrollo del lenguaje y sus características en niños de edad preescolar, por lo que a continuación se presenta un resumen de algunos de los más destacados. En primer lugar se puede indicar — con base en la indagación de Ríos-Flórez, Marulanda, Ruiz y Jiménez (2016) — que no se ha demostrado que el nacimiento prematuro afecte el desarrollo del lenguaje infantil. 


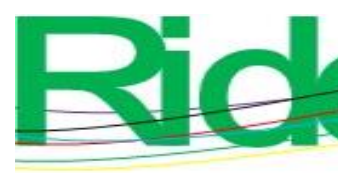

Revista Iberoamericana para la Investigación y el Desarrollo Educativo ISSN 2007 - 7467

Por otra parte, Rojas, Muñoz, Burbano y Pacheco (2019) realizaron un estudio en instituciones colombianas con una muestra de 261 niños con edades de entre 3 y 5 años; los resultados arrojaron alteraciones del lenguaje oral en $46.4 \%$ y la alteración mixta fue la más común con $18.4 \%$, seguida de la alteración del nivel expresivo con $15.3 \%$.

Vasconcelos et al. (2015) en una muestra de 539 estudiantes de entre 4 y 10 años de edad, en Belo Horizonte (Brasil), identificaron que $33.6 \%$ los participantes tenían trastornos del lenguaje oral.

Ilha, Barichello, Rosa de Oliveira, Barichello y Keske (2017), en la ciudad de Santa María (Brasil), evaluaron a 866 niños de 3 a 8 años 11 meses de edad de escuelas privadas y públicas. Sus hallazgos evidenciaron que $26 \%$ de la muestra sufría adquisición fonológica atípica (trastornos fonológicos), lo cual era más latente en alumnos de escuelas públicas.

Samelli, Rondón-Melo, Rabelo y Molini-Avejonas (2017) trabajaron con 479 niños de entre 2 y 5 años de edad pertenecientes a diferentes centros de salud del oeste de Sao Pablo (Brasil). En los resultados hallaron que $26.9 \%$ presentaban déficit en la producción del lenguaje, y $8.6 \%$ en la comprensión del lenguaje.

Schonhaut, Maggiolo, De Barbieri, Rojas y Salgado (2007) —en un estudio realizado en 2006 con niños de 3 a 5 años de edad de la región metropolitana de Chile- detectaron mediante la prueba fonoaudiología que 219 niños (48.8\%) tenían dificultades del lenguaje, mientras que en otra indagación realizada en la misma zona, pero en 2007, hallaron que 36 $\%$ de los participantes también tenían este tipo de problemas linguiísticos (Schonhaut, Maggiolo, Herrera, Acevedo y García, 2008).

Núñez, Granada, Cáceres y Pomés (2017) analizaron la producción y comprensión del discurso en preescolares de la comuna de Talca, séptima región de Chile; para ello, compararon a 20 preescolares con trastorno específico de lenguaje y a 20 alumnos con desarrollo típico. Sus conclusiones demostraron que no había diferencias significativas en la producción de ambos grupos, aunque sí se detectaron en la comprensión del discurso a favor de los niños con desarrollo típico de lenguaje.

Cáceres-Zúñiga, Ramos-Enriquez, Díaz-Gutierrez y Chamorro-Cáceres (2018) —en Talca (Chile) - en una muestra conformada por 112 participantes de nivel preescolar encontraron que $37.5 \%$ sufría de vocabulario receptivo. 


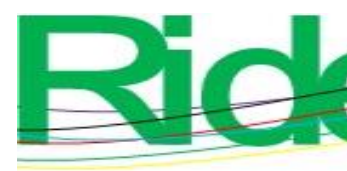

Revista Iberoamericana para la
Investigación y el Desarrollo Educativo
ISSN $2007-7467$

Hurtado-Gamboa y Guerrero-Olalla (2018), al estudiar la prevalencia de retraso en el desarrollo del lenguaje comprensivo y expresivo en 138 niños de 1 a 3 años 11 meses de centros de desarrollo infantil de Ecuador, hallaron retraso del lenguaje comprensivo en 34.7 $\%$ de los participantes, y retraso del lenguaje expresivo en $46.3 \%$ de los alumnos.

Peñafiel-Pinenla y Acosta-Ceballos (2018), en un estudio realizado a 154 niños de 1 a 3 años 11 meses de edad — también de centros de desarrollo infantil de Quito (Ecuador)— , determinaron que la prevalencia de retraso en el lenguaje comprensivo era de $22.08 \%$, y del lenguaje expresivo era de $42.21 \%$.

Quiroz-Arciniega, Quiroz-Arciniega y Ruales-Paredes (2019), en 177 niños de 1 a 3 años 11 meses de edad — de centros de desarrollo infantil de la Provincia Tungurahua (Ecuador) - observaron que $44.89 \%$ presentaba retraso de lenguaje comprensivo, y 71.43 $\%$ de lenguaje expresivo.

Morales-Maza y Ruales-Paredes (2019) en centros de desarrollo infantil de la Provincia Pichincha (Ecuador), examinaron a 57 niños de 1 a 3 años 11 meses de edad, y encontraron que $42.11 \%$ tenían problemáticas en cuanto a lenguaje comprensivo, y $85.96 \%$ en el lenguaje expresivo.

Díaz, Gallestey, Vargas-Machuca y Aguilar Velarde (2017) realizaron una investigación con niños menores de 5 años de Loreto, Ayacucho, Huancavelica y Apurímac (Perú) para identificar la influencia de los factores socioeconómicos en el desarrollo motor y de lenguaje. Los resultados mostraron que los niños de las áreas rurales, hijos de madres con baja escolaridad y pertenecientes a hogares con necesidades básicas insatisfechas exhiben valores más bajos en las dos áreas de desarrollo.

Oré-Quiquia, Tito-Donayre y Villafuerte-Martínez (2018) en un estudio efectuado en colegios mixtos de la ciudad de Lima (Perú) con la prueba PLON-R en 123 niños de 5 a 6 años de edad identificaron a $32.5 \%$ con retraso del desarrollo del lenguaje.

Gómez-Altamirano y Rodríguez-Sánchez (2017) realizaron un estudio con 50 niños de cinco años de edad - 30 de escuelas públicas y 20 de escuelas privadas de la ciudad de Cajamarca (Perú) — para determinar el nivel del lenguaje. Los datos recopilados evidenciaron que $34 \%$ de la población de escuelas públicas tenían un nivel bajo del lenguaje, mientras que en las escuelas privadas el porcentaje se ubicó en $22 \%$. 

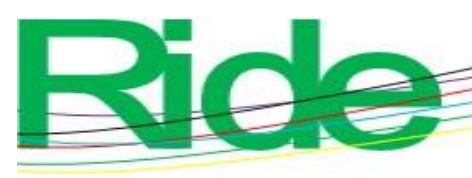

Revista Iberoamericana para la Investigación y el Desarrollo Educativo ISSN 2007 - 7467

Valdivia-Álvarez, I., Gárate-Sánchez, Regal-Cabrera, Castillo-Izquierdo y Sáez (2014), en una investigación desarrollada en La Habana (Cuba) con 45 participantes de 18 meses a 5 años de edad del hospital pediátrico Juan Manuel Márquez — todos con padres de nivel universitario-, hallaron que $80 \%$ de los niños presentó retardo primario del lenguaje.

Eadie et al. (2015) "en un trabajo realizado a 1494 niños australianos con edad de 4 años, encontraron a $40.8 \%$ con trastorno del lenguaje” (p. 578-584). Por su parte, Blumenfeld et al. (2018) evaluaron a 138 participantes de un centro de atención de salud de Argentina. Los resultados demostraron que $11.6 \%$ tenía retraso en el desarrollo del lenguaje.

Granados, Castañeda y Mora (2019) trabajaron con 80 preescolares (32 niñas y 48 niños) con edad promedio de 5 años y 8 meses de escuelas públicas de Xalapa, Veracruz (México). Estos autores evaluaron la inteligencia verbal con la escala Wechsler para los niveles preescolar y primario, además de los signos neurológicos blandos y la narración de una historieta. Los resultados demostraron diferencias significativas en el nivel de coherencia narrativa por dificultades de lenguaje de moderadas a severas.

Finalmente, el Instituto Nacional para la Evaluación de la Educación (INEE) (2011), a nivel nacional en México, identificó a $10 \%$ de la población con insuficiencias en lenguaje y comunicación.

\section{Objetivo general}

El objetivo de este trabajo fue evaluar el lenguaje en niños preescolares del norte de la ciudad de Durango, Durango (México) utilizando el cuestionario de madurez neuropsicológica infantil.

\section{Metodología}

El estudio fue de tipo no experimental, transeccional y descriptivo, ya que se recolectaron datos en un solo momento con el propósito de describir el comportamiento de las variables mediante el uso de la media obtenida (Hernández, Fernández y Baptista, 2014). 


\section{Muestreo}

Para el desarrollo de la investigación se consideró la selección de una muestra probabilística seleccionada aleatoriamente a partir de una población de 833 alumnos de preescolar pertenecientes a la zona norte urbana de la capital del estado de Durango, Durango (México), con un nivel de confianza de $95 \%$ y un margen de error de $5 \%$. La muestra definida mediante la fórmula de población finita — quedó constituida por 264 participantes. Sin embargo, debido a las limitaciones de tiempo por la culminación del ciclo escolar, solo se logró evaluar a 148 participantes. Los criterios elegidos para seleccionar a la muestra fueron los siguientes (Arias, Villasís y Miranda, 2016; Fuentes, 2015):

\section{Inclusión}

1. Niños y niñas de cinco a seis años.

2. Alumnos que cursaban el tercer grado de preescolar.

3. El preescolar debía pertenecer a la zona norte urbana de la capital del estado de Durango.

4. Niños cuyos padres y directivos estuvieran de acuerdo con la evaluación.

\section{Exclusión}

1. Quienes no asistieran el día de la prueba.

2. Edad fuera del rango definido.

3. Niños con alguna discapacidad.

4. Indisposición para realizar la prueba.

\section{Eliminación}

1. Niños cuyos padres no estuvieron en la reunión informativa.

2. Niños cuyos padres no autorizaron la participación en el estudio.

3. Evaluación incompleta. 


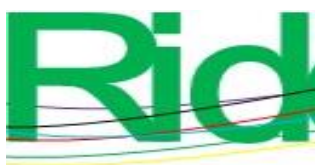

Revista Iberoamericana para la Investigación y el Desarrollo Educativo ISSN $2007-7467$

Universidad Juárez del Estado de Durango. Por último, los recursos físicos fueron 17 salones, 4 mesas, 17 escuelas y 8 sillas.

\section{Procedimiento}

El trabajo se llevó a cabo entre noviembre de 2017 y junio de 2018 en la zona norte urbana de la ciudad de Durango, Durango (México). Las escuelas preescolares que la Secretaría de Educación del Estado de Durango (SEED) tiene registradas en la zona de estudio y el número de participantes evaluados de cada escuela fueron los siguientes (tabla 1):

Tabla 1. Preescolares de estudio

\begin{tabular}{|c|c|}
\hline Escuelas & Participantes a evaluar \\
\hline 1. María Montessori & 8 \\
\hline 2. Gabino Barreda & 7 \\
\hline 3. Diana Laura Rojas Reyes & 9 \\
\hline 4. Martín González Vázquez & 12 \\
\hline 5. Alfonso Reyes & 14 \\
\hline 6. Carmen Serdán a la Triste & 10 \\
\hline 7. León Felipe & 13 \\
\hline 8. José Manuel Puig Causaran & 7 \\
\hline 9. Topiltzin & 12 \\
\hline 10. Amelia Gamero de Ramírez & 6 \\
\hline 11. Fray Diego de la Cadena & 9 \\
\hline 12. Nicolás Bravo & 8 \\
\hline 13. Luis Braille & 7 \\
\hline 14. Justo Sierra & 10 \\
\hline 15. María Luisa Ross Landa & 5 \\
\hline 16. Federico Chopin & 3 \\
\hline 17. Catarino Herrera Barraza & 8 \\
\hline TOTAL & 148 \\
\hline
\end{tabular}

Fuente: Elaboración propia 


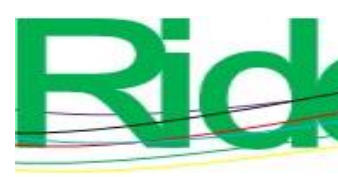

운

Revista Iberoamericana para la Investigación y el Desarrollo Educativo ISSN 2007 - 7467

Una vez identificadas las escuelas participantes, se interactuó primeramente con los directivos de cada institución para solicitar el permiso de realizar el trabajo de investigación. Luego, se solicitó la autorización de los maestros de cada grupo para aplicar el instrumento y, finalmente, se concretó una reunión con los padres de familia, quienes dieron la firma del consentimiento informado para evaluar a la muestra seleccionada.

Los datos se capturaron en una hoja de cálculo de Microsoft Excel, la cual fue empleada para el análisis estadístico de tipo descriptivo, utilizando la media de las puntuaciones directas resultantes en cada una de las 10 variables consideradas en el Cumanin.

\section{Resultados}

Los resultados obtenidos en la puntuación directa del Cumanin mostraron un alfa de Crombach de 0.618 en la muestra de 148 participantes evaluados, de los cuales 68 fueron niñas (43.9\%) y 80 niños (56.1\%), con edades entre los 55 y 78 meses.

La cantidad más pequeña de participantes estudiados estuvo en el grupo de 55-60 meses de edad, mientras que la mayoría de participantes se ubicó en los grupos de 61-66 y 67-72 meses de edad (tabla 2):

Tabla 2. Distribución de edad por meses cumplidos

\begin{tabular}{|c|c|c|c|c|c|c|}
\hline Grupo de edad & Niñas & $\%$ & Niños & $\%$ & Total & $\%$ \\
\hline $55-60$ & 0 & 0.0 & 2 & 1.4 & 2 & 1.4 \\
\hline $61-66$ & 23 & 15.5 & 32 & 21.6 & 55 & 37.2 \\
\hline $67-72$ & 29 & 19.6 & 38 & 25.7 & 67 & 45.3 \\
\hline $73-78$ & 13 & 8.8 & 11 & 7.4 & 24 & 16.2 \\
\hline Total & 65 & 43.9 & 83 & 56.1 & 148 & 100.0 \\
\hline
\end{tabular}

Fuente: Elaboración propia

En el grupo de 55-60 meses de edad, de las 10 variables estudiadas las primeras 5 muestran valores por encima de la media del Cumanin, y 5 con valores por debajo (tabla 3): 


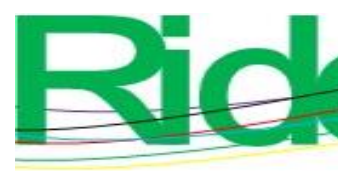

Revista Iberoamericana para la Investigación y el Desarrollo Educativo ISSN 2007 - 7467

Tabla 3. Resultados del grupo de 55-60 meses de edad $(\mathrm{n}=2)$

\begin{tabular}{|l|c|c|c|}
\hline Variables & $\begin{array}{c}\text { Media } \\
\text { obtenida }\end{array}$ & $\begin{array}{c}\text { Porcentaje mayor o menor a la } \\
\text { media del Cumanin }\end{array}$ & $\begin{array}{c}\text { Media del } \\
\text { Cumanin }\end{array}$ \\
\hline $\begin{array}{l}\text { Lenguaje } \\
\text { articulatorio }\end{array}$ & 13 & 19.3 & 10.9 \\
\hline Lenguaje expresivo & 3 & -27.5 & 4.14 \\
\hline Fluidez verbal & 0.5 & -95.9 & 12.06 \\
\hline $\begin{array}{l}\text { Lenguaje } \\
\text { comprensivo }\end{array}$ & 3.5 & -17.6 & 4.25 \\
\hline $\begin{array}{l}\text { Estructuración } \\
\text { espacial }\end{array}$ & 7.5 & 0.1 & 7.49 \\
\hline Psicomotricidad & 9.5 & 28.4 & 7.4 \\
\hline Visopercepción & 7.5 & 11.4 & 6.73 \\
\hline Memoria icónica & 5.5 & 0.4 & 5.48 \\
\hline Ritmo & 1.5 & -34.5 & 2.29 \\
\hline Atención & 6 & -39.5 & 9.91 \\
\hline
\end{tabular}

Fuente: Elaboración propia

En el grupo de 61-66 meses de edad se observan 9 de las 10 variables con valores por debajo de la media del Cumanin, y solo una con resultado por arriba de la media (tabla 4):

Tabla 4. Resultados del grupo de 61-66 meses de edad $(\mathrm{n}=55)$

\begin{tabular}{|l|c|c|c|}
\hline Variables & Media obtenida & Porcentaje de deficiencia & Media del Cumanin \\
\hline Psicomotricidad & 8.2 & 5.1 & 7.8 \\
\hline Lenguaje articulatorio & 9.7 & -18.5 & 11.9 \\
\hline Lenguaje expresivo & 2.7 & -47.1 & 5.1 \\
\hline Fluidez verbal & 2.4 & -86.9 & 18.3 \\
\hline Lenguaje comprensivo & 3.1 & -31.1 & 4.5 \\
\hline Estructuración espacial & 8.2 & -3.5 & 8.5 \\
\hline Visopercepción & 7.1 & -18.4 & 8.7 \\
\hline Memoria icónica & 5.5 & -6.8 & 5.9 \\
\hline Ritmo & 1.9 & -32.1 & 2.8 \\
\hline Atención & 6.7 & -43.2 & 11.8 \\
\hline
\end{tabular}

Fuente: Elaboración propia

En el grupo de 67-72 meses de edad se observan solo 3 variables con valores por arriba de la media, y el resto por debajo de la media del Cumanin (tabla 5): 


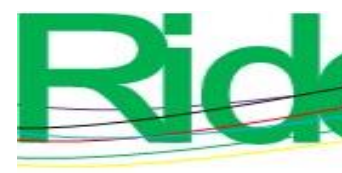

Revista Iberoamericana para la Investigación y el Desarrollo Educativo ISSN 2007 - 7467

Tabla 5. Participantes del grupo de 67-72 meses de edad $(n=67)$

\begin{tabular}{|l|c|c|c|}
\hline Variables & Media obtenida & Porcentaje de deficiencia & Media del Cumanin \\
\hline Estructuración espacial & 9.9 & 13.8 & 8.7 \\
\hline Psicomotricidad & 8.3 & 1.2 & 8.2 \\
\hline Memoria icónica & 6.4 & 1.6 & 6.3 \\
\hline Lenguaje articulatorio & 11 & -14.1 & 12.8 \\
\hline Lenguaje expresivo & 3.2 & -46.7 & 6 \\
\hline Fluidez verbal & 3.8 & -80.5 & 19.5 \\
\hline Lenguaje comprensivo & 4 & -18.4 & 4.9 \\
\hline Visopercepción & 9.3 & -10.6 & 10.4 \\
\hline Ritmo & 3.4 & -2.9 & 3.5 \\
\hline Atención & 7.9 & -41.0 & 13.4 \\
\hline
\end{tabular}

Fuente: Elaboración propia

En el grupo de 73-78 meses de edad solo una variable muestra resultados por arriba de la media, y nueve por debajo de la media del Cumanin (tabla 6):

Tabla 6. Participantes del grupo de 73-78 meses de edad $(n=24)$

\begin{tabular}{|l|c|c|c|}
\hline Variables & Media obtenida & Porcentaje de deficiencia & Media del Cumanin \\
\hline Memoria icónica & 6.6 & 1.5 & 6.5 \\
\hline Lenguaje articulatorio & 11 & -18.5 & 13.5 \\
\hline Lenguaje expresivo & 3 & -55.2 & 6.7 \\
\hline Fluidez verbal & 2 & -90.8 & 21.7 \\
\hline Lenguaje comprensivo & 4.3 & -15.7 & 5.1 \\
\hline Estructuración espacial & 8 & -11.1 & 9 \\
\hline Psicomotricidad & 8 & -3.6 & 8.3 \\
\hline Visopercepción & 8.4 & -25.7 & 11.3 \\
\hline Ritmo & 2.7 & -22.9 & 3.5 \\
\hline Atención & 8.7 & -39.6 & 14.4 \\
\hline
\end{tabular}

Fuente: Elaboración propia

\section{Discusión}

En el presente estudio se evaluó el lenguaje en 148 preescolares de la zona norte de la ciudad de Durango, Durango (México), y aunque la muestra estudiada no fue de 264 - lo que estadísticamente se definió como significativa para el presente estudio-, la confiabilidad de los resultados obtenidos —evaluada con el alfa de Cronbach - fue satisfactoria con 0.618 , lo cual permite realizar un análisis confiable de los resultados.

En los cuatro grupos estudiados se observó a la mayoría de las variables — tanto del área de lenguaje verbal como del área de lenguaje no verbal- con resultados por debajo del valor de la media del Cumanin, punto de referencia para considerar el desarrollo del lenguaje 


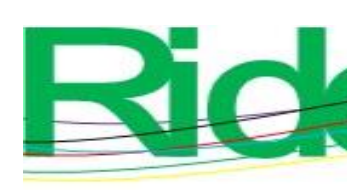

Revista Iberoamericana para la
Investigación y el Desarrollo Educativo
ISSN $2007-7467$

normal. En tal sentido, se destaca en el apartado de lenguaje verbal, la variable fluidez verbal con el valor más bajo, seguida de lenguaje expresivo, lenguaje comprensivo y lenguaje articulatorio. Al considerar que el área del lenguaje verbal es la más afectada, se puede identificar como una razón importante que interfiere con el desarrollo cognitivo de la población preescolar estudiada.

La identificación oportuna del nivel de desarrollo del lenguaje y comunicación oral en los niños constituye una preocupación para padres, maestros y educadores, pues del nivel de desarrollo del lenguaje depende la evolución en la comunicación, el aprendizaje escolar y la personalidad en general (Torres, 2018). Por lo tanto, y observando los resultados de este estudio con porcentajes de deficiencia elevados, se torna importante atender esta problemática que afecta a la población preescolar, como lo expresan Torres (2018), Escobar (2006), Portellano et al. (2006) y Romeo et al. (2018).

\section{Conclusión}

La población estudiada presentó valores por debajo de lo referido como normal por el Cumanin en por lo menos 6 de las 10 variables estudiadas, lo que afecta el desarrollo del lenguaje verbal y no verbal; por ello, se sugiere implementar desde la etapa preescolar estrategias que sirvan para optimizar el lenguaje, herramienta esencial para el desarrollo social y educativo del niño.

La limitación que se tuvo en el trascurso de la investigación estuvo relacionada con la disponibilidad del tiempo, ya que la culminación del ciclo escolar impidió estudiar a la totalidad de la muestra inicialmente definida. Aun así, al haber conseguido un alfa de Cronbach satisfactorio en los resultados obtenidos, se puede indicar que fue confiable el proceso de análisis.

Se recomienda, por tanto, la implementación de evaluaciones del lenguaje sistemáticas desde el inicio y en el transcurso de la educación preescolar del niño, pues de esta manera es factible identificar las necesidades de atención específicas que requiere el infante para un óptimo desarrollo preescolar en cuanto a sus habilidades comunicativas. 


\section{Nuevas líneas de investigación}

Observando los resultados arrojados por el presente trabajo, se logra identificar la necesidad de abrir nuevas líneas de investigación sobre el desarrollo del lenguaje en población preescolar, ya que de esta manera se tendría la oportunidad de mejorar la atención educativa, lo que disminuirá los problemas del lenguaje verbal y no verbal.

\section{Referencias}

Arias, J., Villasís, M. Á. y Miranda, M. G. (2016). El protocolo de investigación III: la población de estudio. Revista Alergia México, 62(2), 201-206.

Blumenfeld, A., Carrizo, J., D’Angelo, S., González, N., Sadras, Y., Graizer, S., Macario, A. y Salamanco, G. (2018). Retraso en el desarrollo del lenguaje en niños de 24 meses en un centro de salud de la Ciudad de Buenos Aires. Archivos Argentinos de Pediatría, 116(4), 242-247. Doi: 10.5546/aap.2018.eng.242

Cáceres-Zúñiga, M. F., Ramos-Enriquez, M., Díaz-Gutierrez, D. y Chamorro-Cáceres, Y. (2018). Vocabulario receptivo en estudiantes de preescolar en la comunidad de Talca, Chile. Innovación Educativa, 18(78), 193-208.

Corral, S. (18 de mayo de 2018). La importancia del lenguaje. Betania Psicología. Recuperado de https://betaniapsicologia.com/2017/05/la-importancia-dellenguaje.html

Díaz, A., Gallestey, J., Vargas-Machuca, R. y Aguilar Velarde, R. (2017). Desarrollo infantil en zonas pobres de Perú. Rev Panam Salud Pública, 41(71). Recuperado de https://iris.paho.org/handle/10665.2/34051:

Eadie, P., Morgan, A., Ukoumunne, O. C., Eecen, K., Wake, M. and Reilly, S. (2015). Speech sound disorder at 4 years: prevalence, comorbidities, and predictors in a community cohort of children. Developmental Medicine \& Child Neurology, 57(6), 578-584.

Escobar, F. (2006). Importancia de la educación inicial a partir de la mediacion de los procesos cognitivos para el desarrollo humano integral. Laurus, 12(21), 169-188.

Fuentes, S. (2015). Cómo se desarrolla un protocolo. Medigraphic, 11(2). Recuperado de https://www.medigraphic.com/pdfs/orthotips/ot-2015/ot152e.pdf

Gómez-Altamirano, K. y Rodríguez-Sánchez, R. D. (2019). Diferencia del lenguaje en niños de 5 años de edad entre una institución de educación inicial pública y privada en la ciudad de Cajamarca (tesis de licenciatura). Perú: Universidad Privada Antonio 


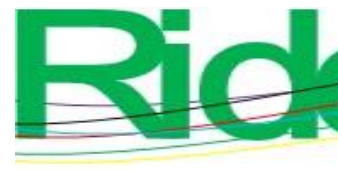

Guillermo
Revista Iberoamericana para la
Investigación y el Desarrollo Educativo
ISSN $2007-7467$

de http://repositorio.upagu.edu.pe/handle/UPAGU/982

Granados, D. E., Castañeda, L. L. y Mora, P. (2019). Análisis de narraciones orales en preescolares con signos neurológicos blandos y dificultades de lenguaje. Lenguaje y Textos, (50). Doi: doi.org/10.4995/lyt.2019.11154

Hernández, S. R., Fernández, C. y Baptista, L. (2014). Metodología de la investigación (6. ${ }^{\text {a }}$ ed.). México: Mc Graw Hill.

Hurtado-Gamboa, M. F. y Guerrero-Olalla, M. P. (2018). Prevalencia de retraso en el desarrollo del lenguaje comprensivo y expresivo en niños de 1 a 3 años 11 meses, de centros de desarrolo infantil, provincia Pichincha (tesis de licenciatura). Ecuador: Universidad Central del Ecuador. Recuperado de http://www.dspace.uce.edu.ec/handle/25000/16662

Ilha, M., Barichello, M., Rosa de Oliveira, C., Barichello, M. and Keske, M. (2017). Prevalence of phonological disorders and phonological processes in typical and atypical phonological development. Codas, 29(3), 1-9.

Instituto Nacional para la Evaluación de la Educación [INEE] (2011). Exámenes de la calidad y el logro educativo. México: INEE.

Morales, A. y Rincón, C. (2016). Relación entre madurez neuropsicológica y presenciaausencia de la conducta de gateo. Acta de Investigación Psicológica, 6(2), 2450-2458.

Morales-Maza, A. D. y Ruales-Paredes, S. S. (2019). Prevalencia de retraso en el desarrollo del lenguaje comprensivo y expresivo en niños de 1 a 3 años 11 meses, en CDI provincia Pichincha ag-2018 a feb-2019 (tesis de licenciatura). Ecuador: Universidad Central de Ecuador. Recuperado de http://www.dspace.uce.edu.ec/handle/25000/17758

Núñez, S., Granada, M., Cáceres, F. y Pomés, M. (2017). Discurso narrativo en preescolares con trastorno específico del lenguaje y con desarrollo típico. Revista Chilena de Fonoaudiología, 16, 1-9.

Oré-Quiquia, D. C., Tito-Donayre, J. A. y Villafuerte-Martínez, L. A. (2018). Retraso del lenguaje en niños de nivel inicial 5 años de colegios emblemáticos mixtos en la ciudad de Lima (tesis de licenciatura). Universidad Peruana Cayetano Heredia. Recuperado de http://repositorio.upch.edu.pe/handle/upch/3805

Peñafiel-Pinenla, B. V. y Acosta-Ceballos, R. E. (2018). Prevalencia de retraso en el desarrollo del lenguaje comprensivo y expresivo en niños de 1 a 3 años 11 meses en 


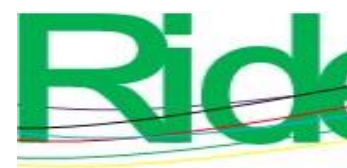

\section{Revista Iberoamericana para la Investigación y el Desarrollo Educativo ISSN $2007-7467$}

centros de desarrollo infantil, provincia Pichincha (tesis de licenciatura). Ecuador: Universidad Central del Ecuador. Recuperado de http://www.dspace.uce.edu.ec/handle/25000/17192

Portellano, J. A., Matreos, R. y Martínez, R. (2006). Cuestionario de madurez neuropsicologica infantil. Madrid: TEA Ediciones.

Quiroz-Arciniega, A., Quiroz-Arciniega, S. y Ruales-Paredes, S. (2019). Prevalencia de retraso en el desarrollo del lenguaje comprensivo y expresivo en niños de 1 año a 3 años 11 meses, en centros de desarrollo Infantil de la provincia Tungurahua (tesis de licenciatura). Ecuador: Universidad Central del Ecuador. Recuperado de http://www.dspace.uce.edu.ec/handle/25000/17830

Ríos-Flórez, J., Marulanda, V., Ruiz, P. y Jiménez, P. (2016). Neuropsicología del lenguaje de niños entre 6 y 10 años de edad con antecedente de nacimiento prematuro. Revista Chilena de Neuropsicología, 11(2), 6-12.

Rojas, L., Muñoz, D., Burbano, E. y Pacheco, R. (2019). Alteraciones del lenguaje oral en niños institucionalizados en hogares comunitarios: prevalencia y determinantes. Interdisciplinary Journal of Epidemiology \& Public Health, 2(1), 1-10.

Romeo, R., Segaran, J., Leonard, J., Robinson, S., West, M., Mackey, A., Yendiki, A., Rowe, M. and Gabrieli, J. (2018). Language Exposure Relates to Structural Neural Connectivity in Childhood. The Journal of Neuroscience, 38(36), 7870 -7877.

Samelli, A., Rondon-Melo, S., Rabelo, S. and Molini-Avejonas, D. R. (2017). Association between language and hearing disorders - risk identification. Clinics, 72(4), 213-217.

Schonhaut, L., Maggiolo, M., De Barbieri, Z., Rojas, P. y Salgado, A. (2007). Dificultades de lenguaje en preescolares: concordancia entre el test TEPSI y la evaluación fonoaudiológica. Revista Chilena de Pediatría, 78(4), 369-375.

Schonhaut, L., Maggiolo, M., Herrera, M. E., Acevedo, K. y García, M. (2008). Lenguaje e inteligencia de preescolares: análisis de su relación y factores asociados. Revista Chilena de Pediatría, 79(6), 600-606.

Torres, N. (2018). Una mirada sobre la prevención de los trastornos del lenguaje y la comunicación oral en los niños de la infancia preescolar. Revista Caribeña de Ciencias Sociales. Recuperado de https://www.eumed.net/rev/caribe/2018/11/prevencion-trastornos-lenguaje.html 

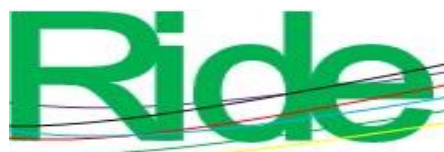

Revista Iberoamericana para la Investigación y el Desarrollo Educativo ISSN $2007-7467$

Valdivia-Álvarez, I., Gárate-Sánchez, E., Regal-Cabrera, N., Castillo-Izquierdo, G. y Sáez, Z. M. (2014). Exposición a televisión y retraso primario del lenguaje en menores de 5 años. Revista Cubana de Pediatría, 86(1), 18-25.

Vasconcelos, A., Rodrigues, F., Passos, C., Vasconcelos, B., De lima, A., Lindgren, C. and Figueiredo, L. (2015). Speech and language disorders in children from public schools in Belo Horizonte. Revista Paulista de Pediatría, 33(4), 453-459. 


\begin{tabular}{|c|c|}
\hline Rol de Contribución & Autor (es) \\
\hline Conceptualización & 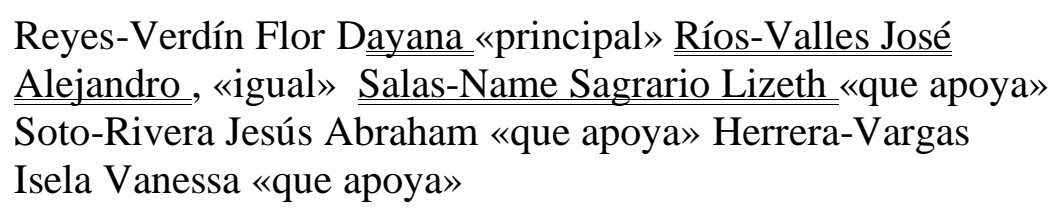 \\
\hline Metodología & $\begin{array}{l}\text { Reyes-Verdín Flor Dayana «principal» } \underline{\underline{\text { Rios-Valles José }}} \\
\text { Alejandro, } \text { «igual» } \underline{\underline{\text { Salas-Name Sagrario Lizeth } « q u e ~ a p o y a » ~}} \\
\text { Soto-Rivera Jesús Abraham «que apoya» Herrera-Vargas } \\
\text { Isela Vanessa «que apoya» }\end{array}$ \\
\hline Software & $\begin{array}{l}\text { Reyes-Verdín Flor Dayana «principal» } \underline{\underline{\text { Ríos-Valles José }}} \\
\text { Alejandro, } \text { «igual» } \underline{\text { Salas-Name Sagrario Lizeth } « q u e ~ a p o y a » ~} \\
\text { Soto-Rivera Jesús Abraham «que apoya» Herrera-Vargas } \\
\text { Isela Vanessa «que apoya» }\end{array}$ \\
\hline Validación & Ríos-Valles José Alejandro \\
\hline Análisis Formal & Ríos-Valles José Alejandro \\
\hline Investigación & Reyes-Verdín Flor Dayana \\
\hline Recursos & 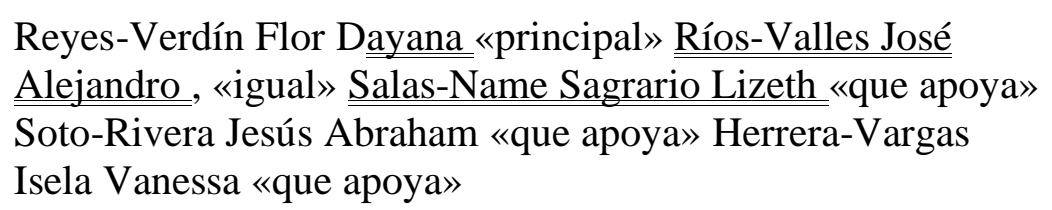 \\
\hline Curación de datos & 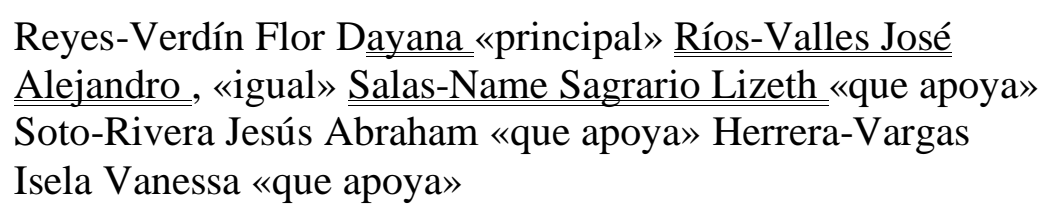 \\
\hline $\begin{array}{l}\text { Escritura - } \\
\text { Preparación del } \\
\text { borrador original }\end{array}$ & $\begin{array}{l}\text { Reyes-Verdín Flor Dayana «principal» } \underline{\underline{\text { Ríos-Valles José }}} \\
\underline{\underline{\text { Alejandro, }}} \text { «igual» }\end{array}$ \\
\hline $\begin{array}{l}\text { Escritura - Revisión } \\
\text { y edición }\end{array}$ & $\begin{array}{l}\text { Reyes-Verdín Flor Dayana «principal» Ríos-Valles José } \\
\text { Alejandro } \\
\text { Soto-Rivera Jesual» Abraham «que apoya» Herrera-Vargas } \\
\text { Isela Vanessa «que apoya» }\end{array}$ \\
\hline Visualización & $\begin{array}{l}\text { Reyes-Verdín Flor Dayana «principal» } \underline{\text { Ríos-Valles José }} \\
\text { Alejandro } \\
\text { Soto-Rivera Jesús A Abraham «que apoya» Herrera-Vargas } \\
\text { Isela Vanessa «que apoya» }\end{array}$ \\
\hline Supervisión & Reyes-Verdín Flor Dayana \\
\hline Administración de & Reyes-Verdín Flor Dayana \\
\hline
\end{tabular}




\begin{tabular}{|c|c|}
\hline & $\begin{array}{l}\text { Revista Iberoamericana para la } \\
\text { Investigación y el Desarrollo Educativo } \\
\text { ISSN } 2007-7467\end{array}$ \\
\hline \multicolumn{2}{|l|}{ Proyectos } \\
\hline $\begin{array}{l}\text { Adquisición de } \\
\text { fondos }\end{array}$ & 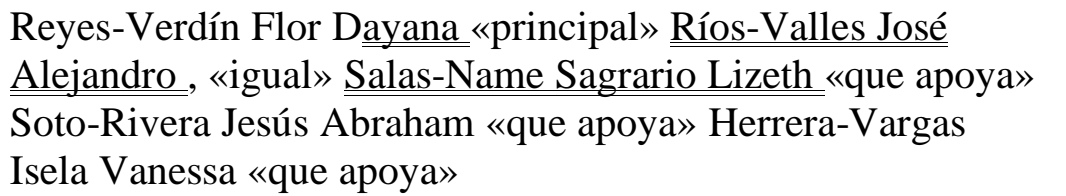 \\
\hline
\end{tabular}

